\title{
Transparent conducting sol gel ATO coatings for display applications by an improved dip coating technique
}

\author{
G. Guzman ${ }^{\mathrm{a}, *}$, B. Dahmani ${ }^{\mathrm{b}}$, J. Puetz $^{\mathrm{c}}$, M.A. Aegerter ${ }^{\mathrm{c}}$ \\ ${ }^{a}$ Corning S.A., Centre Européen de Recherche de Fontainebleau, Avon, France \\ ${ }^{\mathrm{b}}$ Consultant, France \\ ${ }^{\mathrm{c}}$ Leibniz-Institut fuer Neue Materialien- INM, Department of Coating Technology, Saarbruecken, Germany
}

\begin{abstract}
Transparent conducting coatings of sol gel ATO (antimony doped tin oxide) were used to improve surface smoothness of commercial sputter deposited ITO (indium tin oxide) coatings for application as display electrodes. In order to overcome the deteriorating evaporation cooling during dip coating, the coating solution was heated moderately to $25^{\circ} \mathrm{C}$ thus providing the substrate with the required heat. This way, the surface roughness of the ITO could be reduced with an only $45 \mathrm{~nm}$ thick ATO coating to $R_{\mathrm{pv}} 3.8 \mathrm{~nm}\left(R_{\mathrm{a}} 0.4 \mathrm{~nm}\right)$ compared to $31 \mathrm{~nm}$ $(3.8 \mathrm{~nm})$ for the ITO substrate. Another benefit of such additional coating is the possibility to tailor surface properties of the electrodes in wide ranges. This was used to increase the work function of the ITO substrate from initially $4.34 .6 \mathrm{eV}$ to about $4.85 .2 \mathrm{eV}$ by the ATO coating.
\end{abstract}

Keywords: Transparent conducting oxide; Sol gel process; Dip coating; Display

\section{Introduction}

Modern display technology has generated an increasing demand for tailored functional coatings on thin glass substrates - first of all for transparent conducting coatings as a fundamental link between the electrical and optical properties. Though at present the corresponding coatings are mostly deposited by PVD techniques [1,2], sol-gel coatings offer promising options for some forthcoming developments because of their lower deposition cost and superior surface properties. The suitability of these techniques for large area optical coating has already been proven on an industrial scale for the dip coating technique which today is mainly used in the production of antireflection and color effect interference filters $[1,3]$. The ongoing trend towards larger but thinner substrates like Corning Eagle 2000 display glass [4], however, is posing new challenges to meet all coating requirements-both to the coating techniques and the coat- ing materials-and in some cases combinations can help to overcome and solve some of the problems $[5,6]$.

Among the transparent conducting oxide (TCO) coatings, ITO (indium tin oxide, $\operatorname{In}_{2} \mathrm{O}_{3}: \mathrm{Sn}$ ) currently is the material of choice for display applications for its low sheet resistance and the high optical transmission [1,2], but nevertheless commercially available coatings still suffer from some short-comings. Due to the sputtering process such coatings exhibit randomly distributed spikes with a height of up to several tens of nm, which cause problems with electrical break-down as the electrical field strength is drastically increased at the top of such spikes. Another short-coming occurs in applications as electrodes for organic light emitting diodes (OLED), where a hole injection from the ITO layer into the organic light emitting layer is required [7]. For such applications, the work function of ITO, as the fundamental parameter characterizing the ability to inject holes into the emitter layer, is too low with values typically below $4.6 \mathrm{eV}$ whereas work functions larger than 4.8 or even $5.0 \mathrm{eV}$ are desirable. On the other hand, materials with a higher work function than ITO 
all have a lower resistivity and consequently either the sheet resistance of the coating is higher or the thickness has to be increased at the expense of a lower transmission and higher deposition cost.

For the realization of highly transparent coatings with a high electrical conductivity and a high work function, therefore, a combination of several materials and coating techniques was chosen. The low sheet resistance of commercial sputtered ITO substrates should be combined with the coating homogeneity and smoothness of sol-gel ATO coatings, which also have a significantly higher work function than ITO. The following sections report on the challenges that arise from the sol-gel coating of thin displays glass substrates $[8,9]$ and on the achieved coating properties.

\section{Experimental}

Sol-gel coatings of antimony doped tin oxide (ATO, $\mathrm{SnO}_{2}: \mathrm{Sb}$ ) were prepared from an $0.2 \mathrm{M}$ solution of $\mathrm{SnCl}_{2}$ in ethanol with a doping level of $5 \mathrm{~mol}^{\circ} \mathrm{SbCl}_{3}$ and $0.4 \mathrm{M}$ DAA (diacetone alcohol, 4-hydroxy-4-methyl-2-pentanone) as a stabilising agent. Before deposition, the solution was refluxed for $2 \mathrm{~h}$ to reach equilibrium and filtered $(0.2 \mu \mathrm{m})$ to remove larger impurities. Coatings were deposited by the dip coating technique at $4 \mathrm{~mm} / \mathrm{s}$ on different borosilicate glass (AF45-Schott-DESAG, $0.4 \mathrm{~mm}, 1737$-Corning, 0.7 $\mathrm{mm}$, Borofloat 33, Jenaer Glas, $3.3 \mathrm{~mm}$ ) and commercial ITO coated Corning 1737 substrates $\left(0.7 \mathrm{~mm}, \mathrm{R}_{\square}\right.$ : 7-8 $\mathrm{k} \Omega_{\square}$ ) with a size of $7 \times 10 \mathrm{~cm}^{2}$ at constant ambient conditions of $(20 \pm 1)^{\circ} \mathrm{C}$ and $(40 \pm 2) \%$ RH under cleanroom conditions (class 10000) followed by a heat treatment at $550{ }^{\circ} \mathrm{C}$ for $15 \mathrm{~min}$ in air. Coatings were characterized with regards to the thickness by means of a surface profiler after chemical etching (Tencor P10), to the optical haze (Haze-Gard, BYK-Gardner), to the sheet resistance using a linear four-point probe technique and to the work function by means of a Kelvin probe (Au reference) [10]. Investigations of the surface morphology were performed by scanning electron microscopy (SEM, $10 \mathrm{keV}$; Au layer) and by atomic force microscope (AFM, L.O.T.-Oriel NanoView), which was also used to determine the surface roughness. The temperature evolution during dip coating from pure solvents was monitored using an IR thermographical camera (Thermovision 470, AGEMA) [8].

\section{Results and discussion}

\subsection{The issue of evaporation cooling}

First experiments to coat thin display glasses $(0.7 \mathrm{~mm})$ by the dip coating technique as it is commonly used failed and the coatings exhibited a hazy and cloudy appearance as shown in Fig. 1a. In addition, the high reproducibility a)

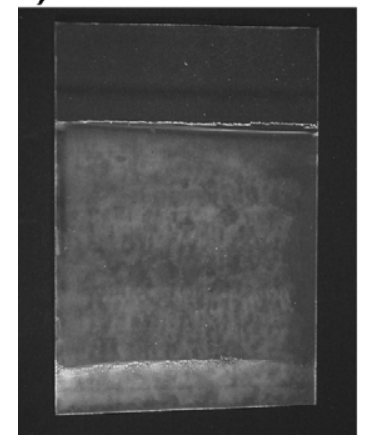

b)

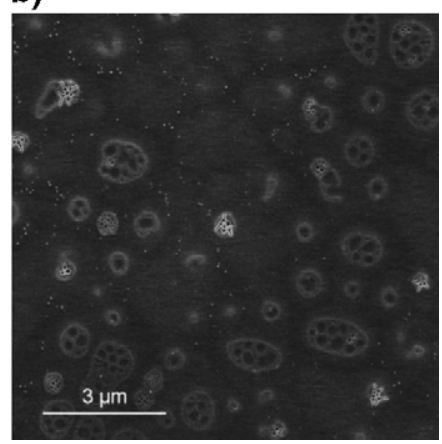

Fig. 1. a) Photograph and b) SEM image of a hazy, cloudy sol gel ATO coating $(\approx 50 \mathrm{~nm})$ on a $0.7 \mathrm{~mm}$ thick 1737 substrate $\left(7 \times 10 \mathrm{~cm}^{2}\right)$ coated by conventional dip coating at room temperature $\left(20^{\circ} \mathrm{C}\right)$.

and pronounced thickness homogeneity of dip coated films could not be achieved by far. A closer examination of the coating morphology revealed lotus pod like crater structures and islands (Fig. 1b) especially in the lower part of the substrate indicating an influence of the solvent evaporation.

This was proofed by means of an IR thermographical monitoring of the early stages of the process of wet film formation during dip coating which has been reported earlier in detail $[8,9]$. According to this investigation, the evaporation of solvents during film drying leads to a significant cooling both of the wet film and of the substrate. The measured temperatures were up to about $12{ }^{\circ} \mathrm{C}$ for 0.4 $\mathrm{mm}$ thick substrates, but almost $18{ }^{\circ} \mathrm{C}$ in the case of the 3 $\mathrm{mm}$ thick substrates. The lower cooling temperature of the thinner substrates arises mainly from their lower heat capacity, as under normal conditions the substrate is the main source of heat for evaporation rather than the surrounding atmosphere.

Though evaporation cooling is a phenomenon common to all wet coating processes, so far it has been largely neglected in the case of dip coating. Its effect on film formation and on the resulting coating quality, however, is tremendous. First of all, the evaporation rate and thus the drying speed are significantly reduced, which in return delays other related and fundamental processes like the hydrolysis and makes them more difficult to control. Moreover, the lower temperature in the surroundings next to the wet film surface also increases the relative humidity and thus increases the available amount of water for hydrolysis - a severe interference in a fundamental step of sol-gel film formation. On very thin substrates $(\leq 0.7 \mathrm{~mm})$ and also in the lower part of the substrate where the solution accumulates, temperatures as low as $8^{\circ} \mathrm{C}$ could be detected, a value which is even below the dew point. But the lowering of the temperature also affects basic rheological properties of the wet film, as for example, its viscosity. In solutions with several liquid components (solvents, stabilizing agents, etc.) the lower temperature also leads to a selective evaporation of more volatile components and this way 
favors a de-mixing of the solution which often leads to wetting problems.

Among the available remedies for this cooling problem, a moderate heating of the coating solution is regarded the most effective, whereas other approaches like the increase of the ambient temperature are little helpful or even deteriorating [8]. The heating of the coating solution is as effective as simple and requires only a low-cost temperature-controlled vessel for the coating solution $\left(20\right.$ to $\left.30{ }^{\circ} \mathrm{C}\right)$. No further change in the usual procedure of dip coating is necessary, besides that the substrate should be kept in the solution for a defined time (typically 30-60 s) before withdrawal to allow temperature equalization. In this way, the required heat for the evaporation during film drying can be stored in the substrate. In practice, an exact compensation of the consumed heat for evaporation is aimed at, as too high solution temperatures on the other hand promote the evaporation from the vessel and the ageing of the solution and also influence the viscosity and the wetting behavior negatively.

The dependence of the coatings properties of the solution temperature is demonstrated in Fig. 2 for a transparent conducting ATO $\left(\mathrm{SnO}_{2}: \mathrm{Sb}\right)$ coating deposited on $0.7 \mathrm{~mm}$ thick Corning 1737 substrates. The characteristic quantities chosen are the haze, as a measure of the optical light scattering and thus of the roughness of the films, and the sheet resistance which characterizes the macroscopic electrical properties and which is extremely sensitive to any change in the composition and the morphology of the coatings. In correspondence, as shown in Fig. 1, the deposition at room temperature $\left(20^{\circ} \mathrm{C}\right)$ leads to a significant increase in the haze of the coatings with a value of up to $8 \%$ and, accordingly, the electrical properties are also worsened. With increasing solution temperature the smoothness and continuity of the coatings increases as indicated by the decreasing haze and sheet resistance. At a temperature of about $25^{\circ} \mathrm{C}$ a minimum is obtained in both quantities with values of approx. $0.1 \%$ for

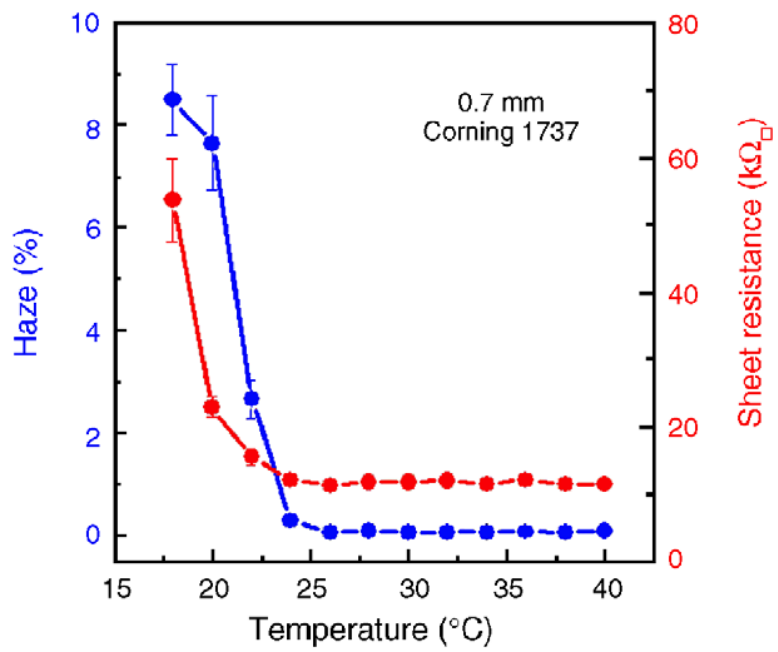

Fig. 2. Sheet resistance and haze of sol gel ATO coatings $(\approx 45 \mathrm{~nm})$ on 0.7 mm thick 1737 substrates in dependence of the temperature of the coating solution. the haze and approx. $12 \mathrm{k} \Omega_{\square}$ for the sheet resistance. This suggests that the heat loss due to evaporation was compensated effectively and that no significant cooling occurred as could be proofed by IR thermographical monitoring. Accordingly, a further increase of the solution temperature beyond $25^{\circ} \mathrm{C}$ is not necessary and should be avoided to minimize evaporation losses.

The heating of the solution, however, does not only help in the coating of thin substrates, but also improves the coating quality on thicker substrates in general and significantly decreases the sensitivity of the wet film against fluctuations of the atmosphere (humidity, temperatures) which otherwise often is a critical parameter.

\subsection{The smoothening effect of sol-gel films}

The modified dip coating technique was used to deposit sol-gel coatings of transparent conducting ATO on commercial ITO substrates (Corning 1737 glass). The intentions behind this additional coating were twofold: first of all, the intrinsic, process-inherent roughness of the low-resistance ITO substrate should be smoothened to avoid electrical break-down and, additionally, the work function of the substrate should be increased without changing the conductivity of the ITO substrate.

The surface morphology of the commercial ITO substrate as revealed by AFM is shown in Fig. 3a on a scale of $10 \times 10 \mu \mathrm{m}^{2}$ (see also Table 1 ). The average roughness $R_{\mathrm{a}}$ of the sample is in the order of $4 \mathrm{~nm}$, but, due to the spikes, the peak-to-valley roughness $R_{\mathrm{pv}}$ takes values of up to $32 \mathrm{~nm}$, causing problems of electrical break-down in some applications. For an effective smoothening of the rough surface, so that the deposited layer should cover the spikes completely, a thickness of about $45 \mathrm{~nm}$ was chosen for the sol-gel ATO layer.

The improved surface morphology of such ATO-coated ITO substrates is shown in Fig. $3 \mathrm{~b}-\mathrm{d}$ on different scales. The complete coverage and smoothening of the ITO surface can be seen in the direct comparison in Fig. 3a and b, though the ATO coated substrate exhibits some larger bumps and irregularities, probably originating from larger defects (e.g. impurities). On a smaller scale of $100 \times 100 \mathrm{~nm}^{2}$, as shown in Fig. 3c and d, the coating surface however is perfectly smooth with an average roughness $R_{\mathrm{a}}$ of only $0.4 \mathrm{~nm}$ and a peak-to-valley roughness $R_{\mathrm{pv}}$ of $3.8 \mathrm{~nm}$. No visible inhomogeneities or protruding spikes from the underlying ITO coating could be detected.

\subsection{The modification of surface properties}

One of the major problems during the dip coating of sputter deposited ITO substrates, however, is the loss of conductivity during high temperature treatment in air, as it is required for sol-gel coatings. On heating the bare ITO substrates in air, the sheet resistance is increasing starting at temperatures of $350{ }^{\circ} \mathrm{C}$ (Fig. 4), due to a loss of oxygen 
a)

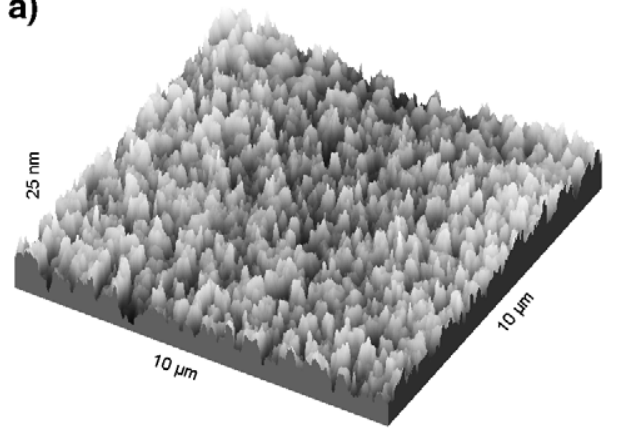

c)

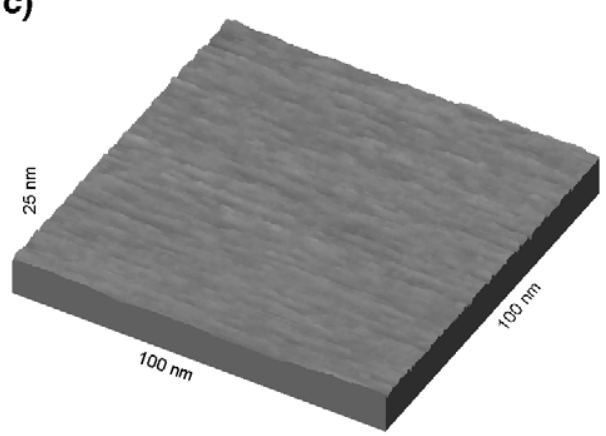

b)

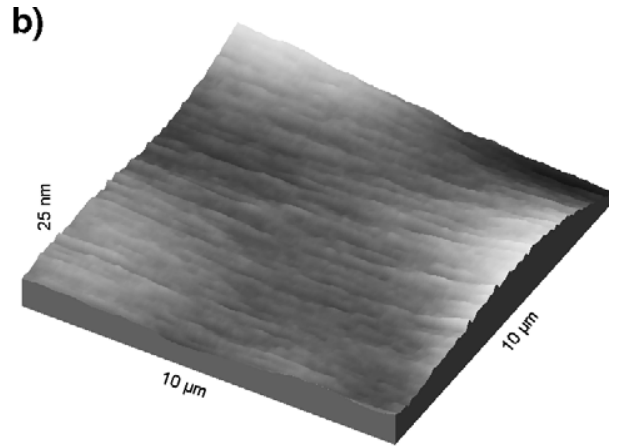

d)

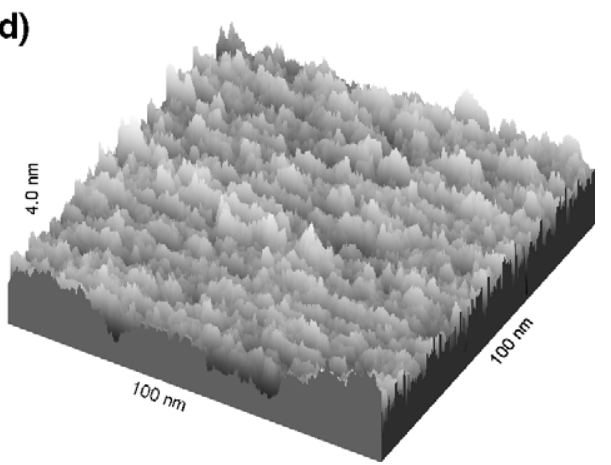

Fig. 3. AFM images of an ITO coated 1737 substrate, a) as-received, b) d) with a dip coated ATO coating (approx. 45 nm) on different scale.

vacancies, which in the case of ITO substantially determine the conductivity. The initial sheet resistance of the sputter deposited ITO substrates of 7 to $8 \Omega_{\square}$ thus increases to more than $60 \Omega_{\square}$ after heat treatment at $550{ }^{\circ} \mathrm{C}$ in air for $15 \mathrm{~min}$, which are common values for the sintering of transparent conducting sol-gel coatings. In the case of the ATO coated ITO substrate, however, the sheet resistance increases only to a minor extent with a final value of $17 \Omega_{\square}$ (cf. Table 1). The additional ATO layer acts like a sealing against oxygen for the ITO coating, by increasing the diffusion path length for oxygen. To further decrease the sheet resistance of the coatings, a post-treatment under inert or reducing atmosphere could be used to partly recover the oxygen vacancies.

Though the sheet resistance of the sol-gel ATO coating is almost 3 orders of magnitude higher than that of the ITO coating, the overall sheet resistance in this parallel arrangement of conductors is largely determined by the highly conductive ITO. For the application, however, it is extremely important to have a conductive coating on top to guarantee an electrical contact, even if the conductivity is

Table 1

Properties of sol gel ATO coatings and ATO coated and bare ITO substrates

\begin{tabular}{|c|c|c|c|c|c|}
\hline Coating & $\begin{array}{l}\text { Thickness } \\
(\mathrm{nm})\end{array}$ & $\begin{array}{l}\text { Sheet } \\
\text { resistance } \\
\left(\Omega_{\square}\right)\end{array}$ & $\begin{array}{l}R_{\mathrm{a}} \\
(\mathrm{nm})\end{array}$ & $\begin{array}{l}R_{\mathrm{pv}} \\
(\mathrm{nm})\end{array}$ & $\begin{array}{l}\text { Work } \\
\text { function } \\
(\mathrm{eV}) \\
\end{array}$ \\
\hline ITO & $192 \pm 2$ & $7.7 \pm 0.1$ & 3.8 & 31 & $4.3 \quad 4.6$ \\
\hline ATO & $45 \pm 1$ & $5.4 \times 10^{3} \pm 21$ & & & $4.8 \quad 5.2$ \\
\hline ATO/ITO & $238 \pm 4$ & $17.0 \pm 0.5$ & 0.4 & 3.8 & $\begin{array}{ll}4.8 \quad 5.2\end{array}$ \\
\hline
\end{tabular}

$R_{\mathrm{a}}$ and $R_{\mathrm{pv}}$ were determined by AFM on a scale of $100 \times 100 \mathrm{~nm}^{2}$. comparatively low. In contrast, an only $40 \mathrm{~nm}$ thick layer of a dielectric like $\mathrm{SiO}_{2}$ would provide a perfect insulation on top of the conductive ITO.

In addition to the smoothening effect of the sol-gel ATO, such materials can also be used to apply other material properties to the highly conductive ITO substrate. The same ATO layer for example was used to increase the work function $\Phi$, as the intrinsic for pure ITO $(4.3-4.6 \mathrm{eV})$ is too low for some applications, especially as electrodes for OLEDs. As the work function is a typical material surface property that is largely independent of the thickness of the material, an improvement could be expected even for very thin layers. Accordingly, the work function of the ATO coated ITO substrates could be increased significantly up to

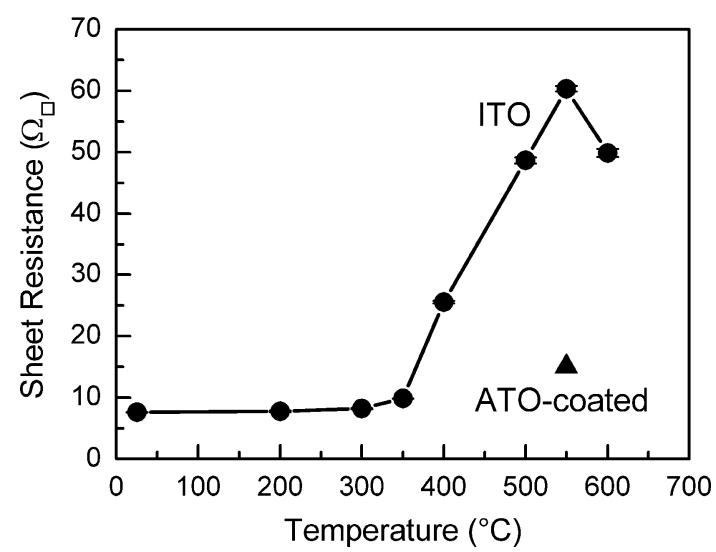

Fig. 4. Sheet resistance of an ITO substrate in dependence of the temperature of heat treatment in air $(15 \mathrm{~min})$. The single data point (triangle) represents the value of an ATO-coated ITO substrate. 
values between 4.8 and $5.2 \mathrm{eV}$, which are sufficient for most applications.

\section{Conclusions}

The combination of different coating materials and deposition techniques offers new possibilities to meet the ever-increasing requirements of coating applications. This has been successfully shown for the sol-gel deposition of ATO on sputter deposited ITO substrates to improve surface smoothness and to tailor surface properties like the work function. The additional ATO coating influences the electrical conductivity of the underlying ITO only little and, on the contrary, protects it against loss of oxygen vacancies during heat treatment. An electrical conductivity of this surface layer, however, is necessary to allow an efficient contacting in application. Therefore, other transparent conducting oxides with even higher work function but lower conductivity as for examples some ternary oxides, can be also of interest.

The sol-gel coating of thin display glasses, however, also poses new challenges to the dip coating procedure, as on the one hand the always present evaporation cooling under normal conditions deteriorates the coating quality, and, on the other hand, the cleanliness becomes a decisive factor. While the evaporation cooling can be controlled effectively by a simple heating of the coating solution, the avoidance of contaminations both in the coating solution and the environment require a more sophisticated control of the deposition process.

\section{References}

[1] H.J. Gläser, Large Area Glass Coating, Von Ardenne Anlagentechnik $\mathrm{GmbH}$, Dresden, 2000 (chapter 4).

[2] H.L. Hartnagel, A.L. Dawar, A.K. Jain, C. Jagadish, Semiconducting Transparent Thin Films, Institute of Physics Publishing, Bristol, 1995, p. 358.

[3] J. Puetz, M.A. Aegerter, Dip coating technique, in: M.A. Aegerter, M. Mennig (Eds.), Handbook on Sol Gel Technologies for Glass Producers and Users, Sol Gel Technologies for Glass Producers and Users, Kluwer Academic Publishers, Boston, 2004, p. 37.

[4] S.T. Gulati, J.D. Helfinstine, J.F. Bayne, J.C. Lapp, SID International Symposium, Baltimore, Maryland, USA, May, 2003.

[5] J.P. Cronin, A. Agrawal, M. Trosky, Method for reducing haze in tin oxide transparent conductive coatings, US5900275, 15.07.1992.

[6] G. Guzman, B. Dahmani, J. Puetz, M.A. Aegerter, Material for use in the manufacturing of luminous display devices WO2004023436A2, 03.09.2003 (see also: FR2844136A1, 03.09.2002).

[7] Y.-H. Tak, K.-B. Kim, H.-G. Park, K.-H. Lee, J.-R. Lee, Thin Solid Films 411 (2002) 12.

[8] J. Puetz, M.A. Aegerter, G. Guzman, J. Sol Gel Sci. Technol. 32 (2004) 125

[9] G. Guzman, J. Pütz, M. Aegerter, A method for depositing a film on a substrate, WO2004022248A1, 03.09.2003 (see also: FR2842899A1, 03.09.2002)

[10] I.D. Baikie, P.J. Estrup, Rev. Sci. Instrum. 69 (1998) 3902. 